\title{
Estrés académico en estudiantes universitarios en contexto de la pandemia por covid-19: una revisión sistemática
}

\author{
Mg. Blas Oscar Sanchez Rasmos \\ Oscarsanchez@unat.edu.pe \\ Universidad Nacional Autónoma de Tayacaja \\ "Daniel Hernández Morillo" \\ Dr. Arnaldo Virgilio Capacha Huamaní \\ arnaldovirgilio365@gmail.com \\ Universidad Nacional de Huancavelica \\ Mg. Mery Luz Capcha Huamaní \\ merychsalud@unh.edu.pe \\ Universidad Nacional Autónoma de Tayacaja \\ "Daniel Hernández Morillo" \\ Dorcas Jeselin Quispe Olano \\ jselin.quispe@gmail.com \\ Universidad Nacional Autónoma de Tayacaja \\ "Daniel Hernández Morillo" \\ Sharoon Zarelly Reza Condori \\ Zarelly@gmail.com \\ Universidad Nacional Autónoma de Tayacaja \\ "Daniel Hernández Morillo"
}

\section{RESUMEN}

Objetivo: El objetivo de este estudio es analizar y determinar el estado de estrés académico que se presenta en los estudiantes universitarios a causa de las clases virtuales en el contexto de la pandemia por COVID19.

Métodos: Se realizó una revisión de información en la base de datos de Google Académico, Scielo, Redalyc, Dialnet y Chemedia entre el mes de diciembre 2020 y parte del mes de agosto 2021. Se evaluó y analizo los resultados, discusión de resultados y conclusiones de los artículos científicos originales, breves, de revisión sistemática, se aplicó el método científico, método deductivo e inductivo, de análisis y síntesis.

Resultados: Un total de 15 estudios fueron incluidos en esta revisión sistemática. La salud mental de estudiantes universitarios se ve repercutida en el estrés académico, debido a las clases virtuales por la pandemia del Covid-19. Aunque existió una amplia 
variabilidad de resultados, se observaron en este sentido un notorio nivel medio y moderado de estrés académico presente en estudiantes universitarios que se evidencia a nivel nacional y mundial.

Conclusiones: La salud mental de los estudiantes universitarios se ven muy comprometidos por la forzosa e inesperada adaptación a llevar clases virtuales en el contexto de confinamiento por la pandemia por la COVID-19, en este sentido es notorio el nivel medio y moderado de estrés académico presente en estudiantes universitarios que se evidencia a nivel nacional y mundial.

Palabra clave: covid-19; estrés académico; estudiantes universitarios; clases virtuales; pandemia; coronavirus; salud mental. 


\title{
Academic stress in university students in the context of the covid-19 pandemic: a systematic review
}

\begin{abstract}
Objective: The objective of this study is to analyze and determine the state of academic stress that is presented in university students due to virtual classes in the context of the COVID19 pandemic.

Methods: An information review was carried out in the Google Academic, Scielo, Redalyc, Dialnet and Chemedia databases between December 2020 and part of August 2021. The results, discussion of results and conclusions of the original, brief scientific articles, of systematic review, the scientific method, deductive and inductive method, of analysis and synthesis was applied.
\end{abstract}

Results: A total of 15 studies were included in this systematic review. The mental health of university students is affected by academic stress, due to virtual classes due to the Covid-19 pandemic. Although there was a wide variability of results, a notorious medium and moderate level of academic stress present in university students was observed in this sense, which is evidenced at a national and global level.

Conclusions: The mental health of university students are highly compromised by the forced and unexpected adaptation to taking virtual classes in the context of confinement due to the COVID-19 pandemic, in this sense the average and moderate level of academic stress present in university students that is evidenced nationally and globally.

Key word: covid-19; academic stress; college students; virtual classes; pandemic; coronavirus; mental health.

Artículo recibido: 05 octubre. 2021 Aceptado para publicación: 02 noviembre 2021 Correspondencia: Oscarsanchez@unat.edu.pe Conflictos de Interés: Ninguna que declarar 


\section{INTRODUCCIÓN}

Actualmente, la situación mundial representa el avance de la denominada primera pandemia global de la historia. Inició en diciembre de 2019 en Wuhan (China), donde se documentaron los primeros casos de neumonías causadas por un nuevo coronavirus (SARS-CoV-2). Debido a la rápida diseminación y al alcance mundial, el 30 de enero del 2020 la Organización Mundial de la Salud (OMS) declaró un estado de emergencia de salud internacional por este brote y el 11 de marzo de 2020 la COVID19, enfermedad causada por el SARS-CoV-2, fue considerada como una pandemia a nivel mundial.(Pérez Abreu et al., 2020)

Las repercusiones que trae consigo la pandemia han sido muy notorios y se han evidenciado hasta el día de hoy, se dan en todos los ambientes en que se desenvuelve el individuo como la económica, social, laboral, educativa y familiar.

Un problema en la salud mental visto en los estudiantes universitarios es el estrés académico a causa de las clases virtuales por la coyuntura en las que el país y el mundo entero se encuentra actualmente, pues el sistema educativo es el que más cambios experimentó, implementando a nivel global la educación virtual (online). Según la UNESCO (2020) en América Latina y el Caribe son 23400000 estudiantes y 1000400 docentes aproximadamente, los involucrados, es decir más del $98 \%$ del total de estudiantes y docentes universitarios. La implementación de nuevas estrategias didácticas a nivel sincrónico y asincrónicas deficiencias en los modelos de educación virtual han repercutido notablemente entre ellos un impacto mental y desgaste emocional denominado estrés académico, debido a la adaptación de la educación a distancia por clases virtuales, asimismo el de afrontar la presión de los maestros, los trabajos académicos, el tiempo compartido con otras actividades como las prácticas profesionales, la investigación, la proyección y extensión universitaria, y la presión de los padres.(Alania-Contreras et al., 2020)

Esta problemática en la educación universitaria, afecta diversos enfoques desde la percepción del estudiante sobre su capacidad para enfrentar exitosamente las exigencias académicas, lo que pueden generar pensamientos y emociones negativas, afectación física como sueño, dolor de cabeza, ansiedad, desconcentración, desorganización y problemas de adaptación.(Gonzalez, 2020) 
El objetivo de este estudio es analizar determinar el estado de estrés que se presenta en los estudiantes universitarios a causa de las clases virtuales en el contexto de la pandemia por COVID19.

\section{METODOLOGÍA}

Se realizó la búsqueda de información en la base de datos de Google Académico, Scielo, Redalyc, Dialnet y Chemedia, para el cual se llevó a cabo un análisis exhaustivo de cada artículo y estudios que indagaron sobre las repercusiones de las clases virtuales en los estudiantes universitarios en el contexto de la pandemia por el brote del SARS-CoV-2, siendo el estrés como principal repercusión en dichos estudiantes a nivel de la Región Huancavelica, a nivel nacional e internacional.

Se utilizó el método Científico, el método deductivo e inductivo, de análisis y síntesis.

\section{SISTEMATIZACIÓN DE RESULTADOS}

En la actualidad existe la necesidad de reflexionar con respeto a lo que ocurre en este escenario de pandemia mundial; algunos países han cerrado temporalmente sus instituciones educativas para contener la propagación del virus. Según la UNESCO (2020), muchas instituciones educativas cerraron sus puertas, causando un impacto disruptivo académico en más del $91.3 \%$ de la población escolar en todo el mundo, esta realidad nos ha mostrado que no hemos estado preparados para afrontar la situación que estamos viviendo.(Revista \& Umch, 2020)

Desde sus inicios, la humanidad ha tenido que pasar por cambios abruptos y repentinos que han sido causa del replanteamiento de sus modelos de vida. El contexto actual, marcado por la existencia de una nueva pandemia mundial, ha alterado en muchas formas la vida cotidiana de los peruanos del siglo XXI. Uno de los sectores más ampliamente afectados es, sin duda, la educación universitaria. Así, la pandemia por la COVID-19 forzó a los centros de educación superior a postergar las clases dictadas presencialmente y a reemplazarlas por semestres netamente virtuales, claro que se resalta la importancia de entrenar a los estudiantes universitarios en nuevas modalidades educativas, en especial en el uso de la tecnología. (Marín Sánchez et al., 2020)

Mucho se ha escrito sobre la creciente educación virtual y sus ventajas en la educación superior. Sin embargo, la mayoría de las universidades peruanas no estaban preparadas para afrontar los desafíos de una educación no presencial. En especial cuando ella fue fruto de una adaptación forzosa. Ella no solo nos recuerda las deficiencias del sistema de 
educación superior, sino que también es oportuna para reformularnos la calidad de la salud mental en el contexto universitario y las nuevas complicaciones que la coyuntura actual acarrea para esta.(No \& Especial, 2020)

Inicialmente se ha encontrado niveles significativamente más altos de ansiedad, depresión y estrés, en comparación con los estudiantes en épocas normales anteriores. Los resultados sugieren que las consecuencias psicológicas debido a la COVID-19 podrían ser graves y abordar el bienestar, manejo emocional y los procesos de afrontamiento, alcanzaría mayor beneficio para la población universitaria con el apoyo de la sociedad, las familias y las universidades.(Juan Guillermo Mansilla Sepúlveda et al., n.d.)

La adaptación de la enseñanza virtual y el estrés que esta genera es un problema causado por la virtualización forzosa de las clases y que afectó directamente a la salud mental de los estudiantes universitarios. En el cuestionario realizado, al preguntar si las dificultades con la tecnología presentada por los docentes los afectaba, la mayoría afirmó que sí (casi el 73\%). Uno de los encuestados señaló que "Los profesores dejan muchos foros y lecturas que deben ser resueltas en un lapso de tiempo muy corto". Es decir, el proceso de adaptación de las nuevas formas de enseñanza tuvo como consecuencia una sobrecarga académica que los estudiantes, al principio, no pudieron sobrellevar. Sobre ello, se observa que un 93, 2\% de la muestra consideró que su carga académica ha aumentado en relación con ciclos anteriores. Por lo tanto, se concluye que existe una relación entre la inexperiencia de la plana docente en metodologías de enseñanza virtual y la sobrecarga en los estudiantes que afectó significativamente su estabilidad mental en donde surgió un nuevo sentimiento de tensión física y emocional a causa del contexto en la que se encuentran, se habla del estrés. Fue tal la presión sentida que una segunda afección apareció, se habla de la ansiedad por el miedo a reprobar el ciclo virtual. Ello, a su vez, podría significar el retiro del ciclo y la deserción universitaria.(Lovón \& Cisneros, 2020) Al estudio de Adaptación del cuestionario de estrés académico SISCO SV al contexto de la crisis por COVID-19, señalan que la población más joven presenta mayores niveles de estrés y no solo por la situación del encierro vivido, sino también, a las clases virtuales, donde mucho de ellos no estuvieron preparados, sumando a la falta equipos tecnológicos en casa, la mala o débil señal de internet, el escaso número de instituciones con plataformas virtuales, docentes entrenados en docencia virtual, carga académica que se tuvo que recuperar por los días de suspensión, porque en un primer momento casi todas 
las instituciones educativas universitarias no estuvieron preparadas para esta pandemia a nivel mundial. (Alania-Contreras et al., 2020)

Debido a la pandemia del Covid-19, los trastornos psicopatológicos más comunes en la población, junto al estrés, son la ansiedad y la depresión, que, por sus características, el proceso de esta enfermedad está acompañado de miedos, angustias y dudas, lo que provoca que los niveles de ansiedad y depresión suelan ser altos, constantes y persistentes en el tiempo respecto a los estudiantes universitarios de medicina.(Pérez Abreu et al., 2020)

El estrés académico asociada a la pandemia por el COVID-19 se está incrementando. Al análisis preliminar de resultados de la Escala de Afrontamiento del Estrés Académico (ACEA) y del Cuestionario de Percepción del Estrés Académico en estudiantes universitarios asociado al Covid-19, muestra una disminución de la motivación y del rendimiento académico con un aumento del estrés con ansiedad y dificultades familiares.(Gonzalez, 2020)

En el informe de las Naciones Unidas "COVID-19 and the Need for Action on Mental Health" se profundiza el panorama de la salud mental de la población universitaria en la pandemia, la depresión afecta a 264 millones de universitarios a nivel global, el suicidio es la segunda causa de muerte entre jóvenes de 15 a 29 años, la ansiedad y la depresión cuestan a la economía global más de un billón de dólares. En este informe se alerta de un probable aumento de sufrimiento por estrés en la población universitaria a causa de las clases virtuales por el contex to de la pandemia.(Huarcaya-Victoria J, 2020)

En una investigación realizada en el Perú con una muestra de 1264 estudiantes universitarios, más de la mitad de ellos, presentan entre 3 y 6 de 6 indicadores de estrés a causa de la realización de clases virtuales por el contexto de la pandemia por el COVID19 , y este mismo número de indicadores de depresión lo presenta el $45 \%$ de la muestra. El $30 \%$ de la muestra presenta entre 3 y 6 indicadores de evitación experimental.(Carlessi Héctor \& Katia Betty, 2020)

El Impacto psicológico en estudiantes universitarios mexicanos por confinamiento durante las fases 2 y 3 en la pandemia de Covid-19 se vio matizado por la presencia de altos indicadores de síntomas (moderados a severos): estrés (31.92\%), problemas para dormir (36.3\%) y ansiedad (40.3\%). En función de los resultados obtenidos en esta investigación, surge la necesidad imperiosa de implementar programas de prevención, 
control y disminución de los efectos psicológicos generados por el confinamiento durante la pandemia de Covid-19 con el objetivo de garantizar el bienestar y la salud mental de los estudiantes universitarios.(González et al., 2020)

El 60\% de los estudiantes encuestados manifiesta tener alteraciones: Ansiedad (29\%), insomnio (19\%), depresión (10\%), mareos 08\% sintió mareos. Es de entender que el deterioro económico y de la salud física, repercute negativamente en la salud psicológica de los estudiantes universitarios, ya que el adaptarse a un ambiente donde no estaban preparados para dar satisfactoriamente clases asíncronas y síncronas.(Cobo-Rendón et al., 2020)

En una reciente investigación de la UNACH, sobre los impactos del COVID-19 comentan respecto al estudio exploratorio se constató el fuerte impacto que el confinamiento ha tenido para la situación vital del estudiantado universitario principalmente en el ámbito psicológico y académico, y la relación existente con la satisfacción vital y la resiliencia, fundamentalmente en su ámbito académico.(Marquina, 2020)

En una investigación que realizaron en Francia encontraron que en términos generales el $40,9 \%$ de los encuestados universitarios tuvieron al menos una experiencia de trastorno mental adverso, un 39\% manifestaron haber tenido síntomas de trastorno de ansiedad, estrés o trastorno depresivo, mientras que un 26,3\% informaron de síntomas de un trastorno relacionado con el trauma y el estrés (TSRD) relacionado con la pandemia y la presencia de las clases virtuales relacionadas con la pandemia por el COVID-19. Por ello, en las circunstancias actuales, puede ser frecuente que, en la mayoría de la población universitaria, se manifieste ansiedad, miedo, tristeza, enfado y/o impaciencia.(Husky, 2021)

Estudios realizados en Ecuador utilizando el cuestionario como metodología , en donde se incluyeron preguntas abiertas, se recogió interesantes opiniones de estudiantes universitarios en cuanto se refiere a la nueva modalidad de clases virtuales por el contexto de la pandemia y como es que ellos la han estado afrontando: "Mis momentos libres se redujeron a estrés mental debido a las muchas horas que pasaba frente a la computadora, lidiando a veces con mala conexión a internet y esforzándome para ayudar en las tareas del hogar", "Esta nueva modalidad de clases virtuales es lo que me ha afectado, pues no es lo mismo que recibir una clase presencial, siento que no estoy aprendiendo todo lo que debería. También siento una tremenda ira hacia la universidad, pues no piensan en los 
estudiantes y no buscan la manera de ayudarnos de verdad, no piensan en que no todos tenemos la mejor situación económica y por otro lado está el estrés que he desarrollado por estar sentado horas frente al computador en mis largas 3 horas de clases programadas o realizando los trabajos que encargan, mis momentos libres han desaparecido".(Trujillo, 2020)

\section{DISCUSIÓN}

El contexto actual en el que nos encontramos, marcado por la existencia de una nueva pandemia mundial, ha alterado y modificado en muchas formas la vida cotidiana de todas las personas a nivel global en pleno siglo XXI. Uno de los sectores más ampliamente afectados por dicha pandemia es, sin duda, la educación universitaria. De una u otra manera la pandemia por la COVID-19 forzó a los centros de educación superior el Perú y el mundo entero a postergar las clases presencialmente y a reemplazarlas por semestres virtuales.

Pero, se omitió un aspecto muy importante; si ya en si la salud mental de las personas se veía afectada por la cuarentena impuesta como respuesta ante el brote de la COVID-19, la salud mental del alumnado en la universidad se ve aún más alterada por una forzosa virtualización de la enseñanza no esperada y menos aún planificada.

La revisión bibliográfica en consenso evidencia la prevalencia del estrés académico en estudiantes universitarios debido a las clases virtuales por la pandemia del Covid-19. Una situación que suele estar afectando a universitarios no solo a nivel nacional, sino que, también a nivel mundial de manera silenciosa y quedando vulnerable su salud física y emocional, así como también el rendimiento académico de futuros agentes del cambio de la sociedad.

En una investigación realizada en el Perú con una muestra de 1264 estudiantes universitarios, más de la mitad de ellos presentan entre 3 de 6 indicadores de estrés a causa de las clases virtuales por el contexto de la pandemia por el COVID-19, esta situación no solo se ve afectando a nuestro país, ya que también se observa la magnitud del problema a nivel mundial, entre ellos un estudio en estudiantes universitarios en México por confinamiento durante las fases 2 y 3 en la pandemia de Covid-19 se vio la presencia de altos indicadores de síntomas (moderados a severos): entre ellas el estrés (31.92\%), problemas para dormir (36.3\%) y ansiedad (40.3\%). La misma situación se evidencio en Francia, a través de encuesta a universitarios, donde un 39\% manifestaron 
haber tenido síntomas de trastorno de ansiedad, estrés o trastorno depresivo, mientras que un $26,3 \%$ informaron de síntomas de un trastorno relacionado con el trauma y el estrés debido a la pandemia.

En tal sentido, sería muy conveniente realizar más estudios de los factores socioemocionales, en particular, y de salud mental, en general, en estudiantes universitarios para contar con información más específica y poder generar protocolos para dar una mejor atención a esta población en cuanto a su salud mental que al no ser tratada oportunamente puede traer consecuencias aún más graves en un futuro no tan lejano.

\section{CONCLUSIONES}

Realizando la revisión sistemática se llegó a las siguientes conclusiones:

1. Los artículos científicos y estudios realizados demuestran que la salud mental de los estudiantes universitarios se ve muy comprometidos por la forzosa e inesperada adaptación a llevar clases virtuales en el contexto de confinamiento por la pandemia por la COVID-19, en este sentido es notorio el nivel medio y moderado de estrés académico presente en estudiantes universitarios que se evidencia a nivel nacional y mundial.

2. En función de los resultados obtenidos en la revisión bibliográfica, surge la necesidad imperiosa de implementar programas de prevención y tratamiento frente al estrés académico en universitarios, que al no ser tomada como un problema de salud mental va degradando silenciosamente la salud física y mental en dichos estudiantes y que en un futuro no tan alejado tendrá graves consecuencias para su salud.

3. Las evidencias científicas en consenso determinan que el estado de estrés en estudiantes universitarios se ve repercutida a causa de las clases virtuales con respecto a la adaptación, limitaciones, el afrontar la presión de los maestros, los trabajos académicos, el tiempo compartido con otras actividades como las prácticas profesionales, la investigación y entre otros.

\section{REFERENCIAS BIBLIOGRAFICAS}

Alania-Contreras, R. D., Llancari-Morales, R. A., Rafaele de La Cruz, M., \& OrtegaRévolo, D. I. D. (2020). Adaptación del cuestionario de estrés académico SISCO SV al contexto de la crisis por COVID-19. Socialium, 4(2), 111-130. https://doi.org/10.31876/sl.v4i2.79

Carlessi Héctor, H. S., \& Katia Betty, M. S. (2020). Investigaciones en salud mental en 
condiciones de pandemia por el COVID-19.

Cobo-Rendón, R., Vega-Valenzuela, A., \& García-Álvarez, D. (2020). Consideraciones institucionales sobre la Salud Mental en estudiantes universitarios durante la pandemia de Covid-19. CienciAmérica, 9(2), 277. https://doi.org/10.33210/ca.v9i2.322

Gonzalez, L. (2020). Asociados a La Pandemia Por Covid-19. Articulo, ix(25), 158-179.

González, N., Tejeda, A., Espinosa, C., \& Ontiveros, Z. (2020). Impacto psicológico en estudiantes universitarios mexicanos por confinamiento durante la pandemia por Covid-19. Scielo, 644(1), 1-17.

Huarcaya-Victoria J. (2020). Consideraciones sobre la salud mental en la pandemia de COVID-19. Revista Peruana de Medicina Experimental y Salud Pública [revista en Internet] 2020 [acceso 11 de setiembre de 2020]; 37(2): 327-334. 37(2), 327334. https://doi.org/10.17843/rpmesp.2020.372.5419

Husky, M. M. (2021). Psiquiatría integral Estrés y ansiedad entre estudiantes universitarios en Francia durante el confinamiento obligatorio del Covid-19. 111.

Juan Guillermo Mansilla Sepúlveda, D., Científico Luiz Alberto David Araujo, E., Europa del Este Aleksandar Ivanov Katrandzhiev, E., Asistente, C., Lic Graciela Pantigoso de Los Santos, P., Carolina Aroca Toloza, D., Bassa Mercado, J., Heloísa Bellotto, D., Nidia Burgos, D., María Eugenia Campos, M., José Francisco Carrera, F., Keri González, M., Guadarrama González, P., Amelia Herrera Lavanchy, M., Cecilia Jofré Muñoz, M., Mario Lagomarsino Montoya, M., Llanos Reyes, C., Mackenbach, W., Rocío del Pilar Martínez Marín, M., ... Stefan Todorov Kapralov, P. D. (n.d.). CUERPO DIRECTIVO Editor OBUCHILE.

Lovón, M., \& Cisneros, S. (2020). Repercusiones de las clases virtuales en los estudiantes universitarios en el contexto de la cuarentena por COVID- 19 : El caso de la PUCP Impact of virtual classes on the university students in the context of COVID-19 quarantine : The case of the PUCP. Propósitos y Representaciones, 8(3).

Marín Sánchez, O., Rodriguez Landauro, A. J., Marín Machuca, O., Marín Sánchez, U., Gamero C., B. E., \& Vértiz Osores, J. J. (2020). Panorama de la pandemia COVID-19. Cátedra $\quad$ Villarreal, $\quad 8(1), \quad 17-23$. 
https://doi.org/10.24039/cv202081758

Marquina, R. (2020). Autopercepción del estrés en aislamiento social en tiempos de covid-19. Revista ConCiencia EPG, 5(1), 85-99.

No, E., \& Especial, N. (2020). Ciencias de la educación Artículo de revisión *. 5(1), 394404. https://doi.org/10.23857/pc.v5i1.1946

Pérez Abreu, M. R., Gómez Tejeda, J. J., Tamayo Velázquez, O., Iparraguirre Tamayo, A. E., \& Besteiro Arjona, E. D. (2020). Alteraciones psicológicas en estudiantes de medicina durante la pesquisa activa de la COVID-19. Medisan, 24(4), 537548.

Revista, E. U., \& Umch, E. (2020). Impacto emocional por COVID-19 en estudiantes universitarios. Un estudio comparativo Emotional Impact of COVID-19 on College Students: A Comparative study. 1(16), 1-14.

Trujillo, P. (2020). Ansiedad, estrés e ira: el impacto del COVID-19 en la salud mental de estudiantes universitarios Anxiety, stress, and anger: the impact of COVID-19 on university students ' mental health. Investigacion \& Desarrollo. 